\title{
Constraints on a possible evolution of mass density power-law index in strong gravitational lensing from cosmological data
}

\author{
R. F. L. Holanda, ${ }^{1,2,3 \star}$ S. H. Pereira ${ }^{4 \star}$ and Deepak Jain ${ }^{5 \star}$ \\ ${ }^{1}$ Departamento de Física, Universidade Federal de Sergipe, 49100-000 Aracaju - SE, Brazil \\ ${ }^{2}$ Departamento de Física, Universidade Federal de Campina Grande, 58429-900 Campina Grande - PB, Brazil \\ ${ }^{3}$ Departamento de Física Teǿrica e Experimental, Universidade Federal do Rio Grande do Norte, 59078-970 Natal - RN, Brazil \\ ${ }^{4}$ Universidade Estadual Paulista (Unesp) - DFQ - Faculdade de Engenharia de Guaratinguetá, 12516-410 Guaratinguetá - SP, Brazil \\ ${ }^{5}$ Deen Dayal Upadhyaya College, University of Delhi, Sector 3, Dwarka, New Delhi 110078, India
}

Accepted 2017 July 13. Received 2017 July 12; in original form 2017 May 18

\begin{abstract}
In this work, by using strong gravitational lensing (SGL) observations along with Type Ia Supernovae (Union2.1) and gamma-ray burst data (GRBs), we propose a new method to study a possible redshift evolution of $\gamma(z)$, the mass density power-law index of strong gravitational lensing systems. In this analysis, we assume the validity of cosmic distance duality relation and the flat universe. In order to explore the $\gamma(z)$ behaviour, three different parametrizations are considered, namely: (P1) $\gamma\left(z_{1}\right)=\gamma_{0}+\gamma_{1} z_{l}$; (P2) $\gamma\left(z_{1}\right)=\gamma_{0}+\gamma_{1} z_{l} /\left(1+z_{l}\right)$; and (P3) $\gamma\left(z_{1}\right)=\gamma_{0}+\gamma_{1} \ln \left(1+z_{1}\right)$, where $z_{1}$ corresponds to lens redshift. If $\gamma_{0}=2$ and $\gamma_{1}=0$, the singular isothermal sphere model is recovered. Our method is performed on SGL sub-samples defined by different lens redshifts and velocity dispersions. For the former case, the results are in full agreement with each other, while a $1 \sigma$ tension between the sub-samples with low $\left(\leq 250 \mathrm{~km} \mathrm{~s}^{-1}\right)$ and high $\left(>250 \mathrm{~km} \mathrm{~s}^{-1}\right)$ velocity dispersions was obtained on the $\left(\gamma_{0}-\gamma_{1}\right)$ plane. By considering the complete SGL sample, we obtain $\gamma_{0} \approx 2$ and $\gamma_{1} \approx 0$ within $1 \sigma$ c.l. for all $\gamma(z)$ parametrizations. However, we find the following best-fitting values of $\gamma_{1}$ : -0.085 ; -0.16 ; and -0.12 for $\mathrm{P} 1, \mathrm{P} 2$ and $\mathrm{P} 3$ parametrizations, respectively, suggesting a mild evolution for $\gamma(z)$. By repeating the analysis with Type Ia Supernovae from Joint Light Analysis compilation, GRBs and SGL systems this mild evolution is reinforced.
\end{abstract}

Key words: gravitational lensing: strong-supernovae: general-distance scale.

\section{INTRODUCTION}

Gravitational lensing phenomenon is one of the most successful predictions of the general relativity theory characterized by a bending of light when it passes close to a massive object. Particularly, two important quantities can be obtained from strong gravitational lensing (SGL) observations: the Einstein radius and time-delay distance. The former depends on the ratio of angular diameter distances (ADD) between lens/source and observer/source, while the second depends on three distances: the ADD between observer and lens; observer and source; and lens and source. Briefly, this effect is caused by the difference in length of the optical paths and the gravitational time dilation for the ray passing through the effective gravitational potential of the lens (Schneider, Ehlers \& Falco 1992; Kochanek, Schneider \& Wambsganss 2004).

Nowadays, SGL observations become a very important tool to measure cosmological parameters. For instance, each ADD in time-

\footnotetext{
*E-mail: holanda@uepb.edu.br (RFLH); shpereira@feg.unesp.br (SHP);
} djain@ddu.du.ac.in (DJ) delay distance is proportional to the inverse of Hubble constant, $H_{0}$. Actually, the possibility of independent determination of $H_{0}$ using time delay between images was suggested in 1964 by Refsdal, however, only recently the technique has been competitive with other cosmological tests considering a flat $\Lambda$ cold dark matter (CDM) scenario (Saha et al. 2006; Coe \& Moustakas 2009; Suyu et al. 2010, 2013). When combined with cosmic microwave background power spectrum, time-delay distance measurements are very effective at breaking degeneracies such as those between $H_{0}$ and $\omega$, the dark energy equation-of-state parameter (see also excellent reviews in Kochanek, Schneider \& Wambsganss 2004 and Treu 2010). As a new approach, Paraficz \& Hjorth (2009) showed that the ADD to lens can be obtained from a joint analysis between the gravitationally lensed quasar images and dispersion velocity of the lensing galaxy (see also Jee, Komatsu \& Suyu 2015 and Holanda 2016).

The Einstein radius measurement is insensitive to Hubble constant since it is a ratio between two ADD. However, this quantity has been largely used to constrain the cosmological parameters of several models (Futamase \& Yoshida 2001; Biesiada 2006 and Grillo, Lombardi \& Bertin 2008). An expressive work has been done recently by Cao et al. in which concerns applications of SGL data 
(Cao \& Liang 2011; Cao et al. 2015a, 2016a, 2016b) including statistical analyses of observed image separations (Cao \& Zhu 2012), lens redshifts (Cao et al. 2012) and more recently to test postNewtonian models of gravity at galaxy scale (Cao et al. 2017a; see also Mitchell et al. 2005 and Ofek et al. 2003 for additional applications). SGL systems were also used to constrain the cosmic equation-of-state parameter in XCDM cosmology and in the Chevalier-Polarski-Linder (CPL) parametrization, where $\omega$ is allowed to evolve with redshift as $\omega(z)=\omega_{0}+\omega_{1} \frac{z}{1+z}$. Particularly, Cao et al. (2015a) used 118 SGL systems from the Sloan Lens ACS Survey, BOSS emission-line lens survey, Lens Structure and Dynamics and Strong Lensing Legacy Survey, improving the confidence regions on the parameter space. These authors also showed that the analyses with SGL may be complementary to type Ia Supernovae (SNe Ia) data. Very recently, SGL measurements have also been used joined with $\mathrm{SNe}$ Ia observations to test the so-called cosmic distance duality relation (CDDR), $D_{L} D_{A}^{-1}(1+z)^{-2}=1$, where $D_{A}$ is the ADD and $D_{L}$ is the luminosity distance in a given redshift (Liao et al. 2016; Holanda, Busti \& Alcaniz 2016; Holanda, Busti, Lima \& Alcaniz 2016). No significant departure from the CDDR validity with this data set was verified.

However, some problems arise when one uses SGL observations as cosmological tool, for instance, different values of $H_{0}$ are obtained from system to system. In this context, Suyu et al. (2010, 2013) obtained a value of $H_{0}=70.6 \pm 3.1 \mathrm{~km} \mathrm{~s}^{-1} / \mathrm{Mpc}$ for $\mathrm{B} 1608+656$ system and $H_{0}=78.7_{-4.5}^{+4.3} \mathrm{~km} \mathrm{~s}^{-1} / \mathrm{Mpc}$ for RXJ11311231. Another important uncertainty source is the lens mass model, as different values of $H_{0}$ are obtained if one assumes either a singular isothermal spherical profile (SIS model, where $\rho \propto r^{-2}$ ) or a spherically symmetric power-law mass distribution $\left(\rho \propto r^{-\gamma}\right)$. The SIS profile has been widely used to describe lens galaxies, however, several studies have shown that the slopes of density profiles of individual galaxies show a non-negligible scatter from the SIS model (Koopmans 2005; Koopmans et al. 2009; Auger et al. 2010; Barnabe et al. 2010; Sonnenfeld et al. 2013). Moreover, by using 11 early-type galaxies, Ruff et al. (2011) found a mild evolution when the $\gamma$ parameter was allowed to vary with redshift, which would indicate that dissipative processes play some role in the growth of massive galaxies. In other words, a $\gamma$ evolution may play a crucial role on galaxy structures. This fact has been investigated considering SGL observations and complementary probes in some cosmological scenarios, such as: $\Lambda \mathrm{CDM}, \mathrm{XCDM}$ and $X(z) \mathrm{CDM}$ (Cao et al. 2015a; Li et al. 2016; Cui, Li \& Zhang 2017). By using a relation such as $\gamma(z)=\gamma_{0}+\gamma_{1} z$, no significant evidence for the evolution of $\gamma$ from SGL observation has been found. Very recently, by taking the Planck best-fitted cosmology, Cao et al. (2016a) considered SGL observations and relaxed the assumption that stellar luminosity and total mass distribution follows the same power law. Interestingly, they found that the presence of dark matter in the form of a mass component is distributed differently from the light (see also Schwab et al. 2010). Their results also suggested the need of treating low, intermediate and high-mass galaxies separately. At this point, it is very important to stress that the results of these previous studies were obtained by using some specific cosmological model in their analyses. ${ }^{1}$
The main aim of this work is to perform constraints on some $\gamma(z)$ parametrizations without explicitly using any cosmological model. From a theoretical point of view, only a flat universe and the validity of the CDDR relation are assumed. As data sets, we use SGL observations plus SNe Ia and GRBs. In order to access the cosmic history of $\gamma(z)$, our method is applied on two SGL subsamples defined by the velocity dispersions of lenses ${ }^{2}\left(\sigma_{\text {ap }}\right)$ and three SGL sub-samples defined by lens redshifts (see Section 4 for details). Three simple parametrizations for $\gamma(z)$ are proposed, namely: (P1) $\gamma\left(z_{1}\right)=\gamma_{0}+\gamma_{1} z_{1}$; (P2) $\gamma\left(z_{1}\right)=\gamma_{0}+\gamma_{1} z_{1} /\left(1+z_{1}\right)$; and (P3) $\gamma(z)=\gamma_{0}+\gamma_{1} \ln \left(1+z_{1}\right)$, where $z_{1}$ corresponds to lens redshift. It obtained a $1 \sigma$ tension on the $\left(\gamma_{0}-\gamma_{1}\right)$ plane from the results by using the sub-samples with high $\left(>250 \mathrm{~km} \mathrm{~s}^{-1}\right)$ and low $\left(\leq 250 \mathrm{~km} \mathrm{~s}^{-1}\right)$ velocity dispersions. On the other hand, the results from the three sub-samples defined by lens redshifts are in full agreement with each other. We also perform analyses with the complete SGL sample. As we shall see, for those accepting the strict validity of the standard CDDR relation, our analyses suggest no significant departure from a $\gamma\left(z_{1}\right)$ constant, but a mild evolution is allowed by the data.

The paper is organized as follows. In Section 2 we present the methodology, Section 3 contains the data of strong lensing used in our analyses, Section 4 presents the analyses and results and the conclusions are given in Section 5.

\section{METHODOLOGY}

In this section we discuss the key aspects of our methodology, such as: the validity of CDDR, SGL observations (Einstein radius, SIS and power-law models); and various parametrizations of $\gamma(z)$.

\subsection{The cosmic distance duality relation validity}

The main point of our methodology is to consider the validity of the CDDR relation, namely: $D_{L} D_{A}^{-1}(1+z)^{-2}=1$. The so-called CDDR is the astronomical version of the reciprocity theorem proved long ago by Etherington (1933) and it requires only that source and observer are connected by null geodesics in a Riemannian space-time and that the number of photons are conserved (see also Ellis 1971, 2007). It plays an essential role in cosmological observations and has been extensively applied by several authors in different cosmological context (Bassett \& Kunz 2004; Cunha, Marassi \& Santos 2007; Zhu et al. 2008; Cao \& Liang 2011; Holanda, Lima \& Ribeiro 2011; Mantz et al. 2014; Cao et al. 2016b; Rana et al. 2016). Recently, several ways to test this relation have been proposed using different astronomical quantities, such as: SNe Ia plus $H(z)$ data; gas mass fractions; and angular diameter distances of galaxy clusters plus SNe Ia, gamma-ray burst plus $H(z)$, SNe Ia plus barion acoustic oscillations (BAO), cosmic microwave background radiation (CMB), gas mass fraction plus $H(z)$ data, SNe Ia plus CMB plus BAO and gravitational lensing plus SNe Ia. An interesting summary with several results can be found in Table I of Holanda, Busti \& Alcaniz (2016). As a main conclusion, no significant departure from the validity of the CDDR has been verified.

\footnotetext{
${ }^{1}$ There are other important uncertainty sources in the SGL science, such as: velocity anisotropy, mass along the line of sight, the mass-sheet degeneracy and the environment of the lenses. However, in this paper, we are considering only the mass profile shape.
}

\footnotetext{
${ }^{2}$ The dynamical mass is related to the velocity dispersion through the relation $M \propto \sigma_{\text {ap }}^{2}$ in the singular isothermal sphere model (Longair 1998). Thus, one may consider these sub-samples as being divided by lens masses.
} 


\subsection{Einstein radius}

An important measurement used in our analyses is the Einstein radius. When the source (s), the observer (o) and the lens (1) in a SGL system are nearly aligned with each other then a ring like structure is formed called the Einstein radius (Schneider, Ehlers \& Falco 1992; Kochanek, Schneider \& Wambsganss 2004). This quantity depends on the evolution of the strong lensing system and on its mass distribution model. For the simplest one, based on SIS model, the Einstein radius is given by

$\theta_{\mathrm{E}}=4 \pi \frac{D_{A_{\mathrm{Is}}}}{D_{A_{\mathrm{s}}}} \frac{\sigma_{\mathrm{SIS}}^{2}}{c^{2}}$,

where $\sigma_{\text {SIS }}$ is the dispersion velocity due to lens mass distribution, $c$ the speed of light, $D_{A_{\mathrm{I}}}$ and $D_{A_{\mathrm{s}}}$ are the angular diameter distances between lens and source, and observer and source, respectively.

As commented earlier, several studies have shown that slopes of density profiles of individual galaxies exhibit a non-negligible scatter from the SIS model. In this way, the SIS model was generalized in order to assume a spherically symmetric power-law mass distribution of type $\rho \sim r^{-\gamma}$ (which becomes a SIS model for $\gamma=2$ ). So, the Einstein radius is written as (Cao et al. 2015a)

$\theta_{\mathrm{E}}=4 \pi \frac{D_{A_{\mathrm{ls}}}}{D_{A_{\mathrm{s}}}} \frac{\sigma_{\mathrm{ap}}^{2}}{c^{2}}\left(\frac{\theta_{\mathrm{E}}}{\theta_{\mathrm{ap}}}\right)^{2-\gamma} f(\gamma)$,

where $\sigma_{\text {ap }}$ is the stellar velocity dispersion inside an aperture of size $\theta_{\text {ap }}$ and

$f(\gamma)=-\frac{(5-2 \gamma)(1-\gamma)}{\sqrt{\pi}(3-\gamma)} \frac{\Gamma(\gamma-1)}{\Gamma(\gamma-3 / 2)}\left[\frac{\Gamma(\gamma / 2-1 / 2)}{\Gamma(\gamma / 2)}\right]^{2}$.

Therefore, ${ }^{3}$

$D \equiv D_{A_{\mathrm{ls}}} / D_{A_{\mathrm{s}}}=\frac{c^{2} \theta_{\mathrm{E}}}{4 \pi \sigma_{\mathrm{ap}}^{2}}\left(\frac{\theta_{\mathrm{ap}}}{\theta_{\mathrm{E}}}\right)^{2-\gamma} f^{-1}(\gamma)$.

As we discuss further, such generalization jointly with the CDDR validity allows to study models where the mass profile evolves with redshift, namely $\gamma=\gamma(z)$.

\subsection{Investigating the $\gamma(z)$ cosmic evolution with the CDDR validity}

Previous papers proposed to test the CDDR validity by using ADD from SGL systems jointly with luminosity distances from $\mathrm{SNe}$ Ia data (Liao et al. 2016; Holanda, Busti \& Alcaniz 2016; Holanda, Busti, Lima \& Alcaniz 2016). Particularly, in Liao et al. (2016) the method did not depend on assumptions concerning the details of a cosmological model and only a flat universe was assumed. In such flat universe the comoving distance between lens and source, $r_{\mathrm{ls}}$, is given by (Bartelmann \& Schneider 2001)

$r_{\mathrm{ls}}=r_{\mathrm{s}}-r_{1}$.

By using the basic definitions $r_{\mathrm{s}}=\left(1+z_{\mathrm{s}}\right) D_{A_{\mathrm{s}}}, r_{1}=\left(1+z_{1}\right) D_{A_{1}}$ and $r_{\mathrm{ls}}=\left(1+z_{\mathrm{s}}\right) D_{A_{\mathrm{ls}}}$, it is possible to find

$D=1-\frac{\left(1+z_{1}\right) D_{A_{1}}}{\left(1+z_{\mathrm{s}}\right) D_{A_{\mathrm{s}}}}$

\footnotetext{
${ }^{3}$ A more general expression can be obtained if one relaxes the assumption that the stellar luminosity and total mass distribution follows the same power law (see equation (11) in Cao et al. 2016a).
}

In our case, we assume the CDDR validity and the above expression can be written as

$D=1-\frac{D_{L_{1}}\left(1+z_{\mathrm{s}}\right)}{D_{L_{\mathrm{s}}}\left(1+z_{1}\right)}$

Thus, $D$ defined as in above equation depends only on luminosity distances, more precisely, on the luminosity distances to lens and sources of SGL systems. In this work, these quantities are calculated by using $\mathrm{SNe}$ Ia and GRBs data (details are given in Section 4). On the other hand, the same $D$ can also be calculated by using equation (4) from the SGL data. In this case, the only unknown factor is $\gamma(z)$ which can be further parametrized as follows:

(i) P1: $\gamma\left(z_{1}\right)=\gamma_{0}+\gamma_{1} z_{1}$;

(ii) P2: $\gamma\left(z_{1}\right)=\gamma_{0}+\gamma_{1} z_{1} /\left(1+z_{1}\right)$; and

(iii) P3: $\gamma\left(z_{1}\right)=\gamma_{0}+\gamma_{1} \ln \left(1+z_{1}\right)$.

The parametrizations P2 and P3 have not been explored so far in the literature.

\section{DATA}

The following data sets are used in this paper:

\subsection{Angular diameter distances}

(i) The original SGL data comprises 118 systems from Cao et al. (2015a) and were observed in the Sloan Lens ACS survey (SLACS), BOSS Emission-Line Lens Survey (BELLS), Lenses Structure and Dynamics Survey (LSD) and Strong Legacy Survey SL2S, with redshift ranges: $0.075 \leq z_{1} \leq 1.004$ and $0.20 \leq z_{\mathrm{s}} \leq 3$.60. In Table 1 of Cao et al. (2015a), all relevant information necessary to obtain $D$ as defined in equation (4) is displayed.

\subsection{Luminosity distances}

(i) The main $\mathrm{SNe}$ Ia data set used here is taken from Suzuki et al. (2012), which comprises 580 points called Union2.1 compilation. The SNe Ia redshift range is $0.015 \leq z \leq 1.42$. As is largely known, the distance modulus of Union2.1 compilation was calibrated using the SALT II light-curve fitter (Guy et al. 2007). Modern fitters as SALT II calibrate cosmological parameters together with light-curve parameters. That is, the distance modulus is given by $\mu=5 \log \left(D_{L}\right)+25=m_{B}-M+\alpha x-\beta c$, where $M$ is the absolute magnitude, $m_{B}$ is the apparent magnitude, $\alpha$ is the stretch parameter, $\beta$ is the colour parameter and $x$ and $c$ are parameters measured from the light curve. No calibration with local objects is performed. The values of distance moduli used in our analyses were calibrated by using an underlying cosmological model, namely, the flat $\Lambda \mathrm{CDM}$. However, as the Union2.1 consists of several sub-samples, Suzuki et al. (2012) fit a different $M$ for each sub-sample thereby making the impact of the cosmological model very small (see section 4.4 of their paper). Thus, we believe that the Union2.1 sample is sufficient to turn our analyses weakly dependent on a specific cosmological model. We also added quadratically a 0.15 magnitude error, which can be associated with the intrinsic dispersion of all SNe Ia data.

(ii) Since several sources of SGL systems lie in the interval $1.4 \leq$ $z_{\mathrm{s}} \leq 3.6$, i.e. beyond the redshift range of current SNe Ia compilations $(z \approx 1.50)$, we consider also the latest GRBs distance modulus data, whose redshift range is $0.033 \leq z \leq 9.3$. The complete sample from Demianski et al. (2017) has 167 GRBs. These authors used a local regression technique jointly with SNe Ia luminosity distances 
Table 1. Results obtained for the parameters $\gamma_{0}$ and $\gamma_{1}$ for each parametrization P1, P2 and P3 in different ranges of redshift $z_{1}$ and $\sigma_{\text {ap }}(2$ free parameters). All intervals are at $1 \sigma$ c.l..

\begin{tabular}{|c|c|c|c|c|c|c|}
\hline & \multicolumn{2}{|c|}{ P1 } & \multicolumn{2}{|c|}{$\mathrm{P} 2$} & \multicolumn{2}{|c|}{ P3 } \\
\hline & $\gamma_{0}$ & $\gamma_{1}$ & $\gamma_{0}$ & $\gamma_{1}$ & $\gamma_{0}$ & $\gamma_{1}$ \\
\hline$z_{1}<0.2$ & $2.11 \pm 0.20$ & $-0.51 \pm 1.32$ & $2.12_{-0.22}^{+0.23}$ & $-0.67_{-1.83}^{+1.90}$ & $2.11_{-0.24}^{+0.22}$ & $-0.59_{-1.58}^{+1.97}$ \\
\hline $0.2<z_{1}<0.45$ & $2.10 \pm 0.25$ & $-0.33 \pm 0.75$ & $2.13_{-0.27}^{+0.33}$ & $-0.59_{-1.11}^{+0.99}$ & $2.11_{-0.27}^{+0.29}$ & $-0.44_{-1.05}^{+0.99}$ \\
\hline$z_{1}>0.45$ & $2.05 \pm 0.41$ & $-0.065 \pm 0.80$ & $2.07 \pm 0.67$ & $-0.16 \pm 1.95$ & $2.05 \pm 0.54$ & $-0.09_{-1.03}^{+1.10}$ \\
\hline$\sigma_{\mathrm{ap}}<250 \mathrm{~km} \mathrm{~s}^{-1}$ & $2.04_{-0.06}^{+0.08}$ & $-0.03 \pm 0.23$ & $2.05_{-0.09}^{+0.11}$ & $-0.065 \pm 0.44$ & $2.04_{-0.09}^{+0.10}$ & $-0.05_{-0.30}^{+0.31}$ \\
\hline$\sigma_{\mathrm{ap}}>250 \mathrm{~km} \mathrm{~s}^{-1}$ & $1.89_{-0.09}^{+0.29}$ & $0.14_{-0.64}^{+0.34}$ & $1.87_{-0.12}^{+0.43}$ & $0.27_{-1.45}^{+0.48}$ & $1.88_{-0.10}^{+0.42}$ & $0.22_{-0.97}^{+0.43}$ \\
\hline Union $2.1+\mathrm{GRB}+\mathrm{SGL}$ (92 data points) & $2.04_{-0.06}^{+0.08}$ & $-0.085_{-0.18}^{+0.21}$ & $2.05 \pm 0.10$ & $-0.16_{-0.34}^{+0.36}$ & $2.04 \pm 0.11$ & $-0.12_{-0.27}^{+0.29}$ \\
\hline JLA+GRB+SGL (87 data points) & $2.04_{-0.06}^{+0.08}$ & $-0.13_{-0.20}^{+0.19}$ & $2.06 \pm 0.10$ & $-0.26_{-0.35}^{+0.31}$ & $2.05 \pm 0.11$ & $-0.19_{-0.29}^{+0.26}$ \\
\hline
\end{tabular}
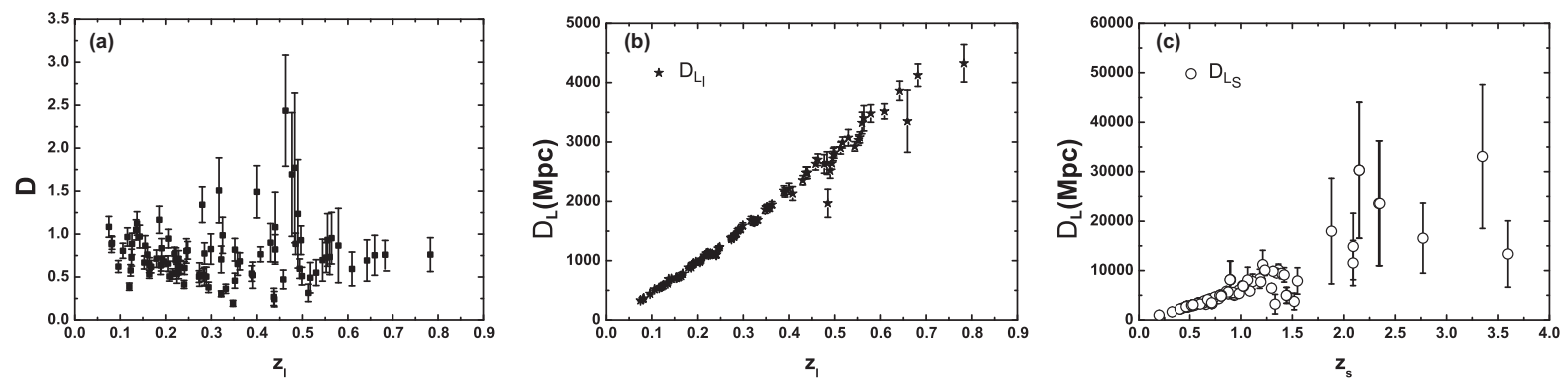

Figure 1. Fig. (a) shows the complete SGL sample (92 points) used in our analyses (considering $\gamma_{0}=2$ and $\gamma_{1}=0$ ). The original SGL sample with 118 points can be found in Cao et al. (2015a). Figs (b) and (c) show the luminosity distances to the lenses (filled star) and to the sources (open circles), respectively, of the 92 SGL systems in the Fig. (a). These luminosity distances were obtained from original SNe Ia (Suzuki et al. 2012) and gamma-ray burst (Demianski et al. 2017) data.

(Union2.1) to calibrate several correlations between spectral and intensity properties, which suggest that GRBs can be used as distance indicators. Moreover, no dependence on redshift of the correlations were found.

\section{ANALYSES AND RESULTS}

In order to perform the analyses with equation (7) we need luminosity distances to the lens and source of each SGL system. These quantities are obtained as follows: for each one of the 118 SGL systems, we carefully select SNe Ia and GRBs with redshifts obeying the criteria $^{4}$ (I) $\left|z_{1}-z_{\mathrm{SNe} / \mathrm{GRB}}\right| \leq 0.006$ and (II) $\left|z_{\mathrm{s}}-z_{\mathrm{SNe} / \mathrm{GRB}}\right| \leq$ 0.006 . Obviously, the SNe Ia or GRBs obeying (I) and (II) are not the same. Finally, we calculate the following weighted average for the distance modulus selected in each case

$\bar{\mu}=\frac{\sum\left(\mu_{i} / \sigma_{\mu_{i}}^{2}\right)}{\sum 1 / \sigma_{\mu_{i}}^{2}}, \quad \sigma_{\bar{\mu}}^{2}=\frac{1}{\sum 1 / \sigma_{\mu_{i}}^{2}}$.

After all, we end with a sample containing 92 SGL systems (see Fig. 1a) and $184 \bar{\mu}$ from SNe Ia (Union2.1) and GRBs data (two $\bar{\mu}$ for each SGL system are necessary). Naturally, $\bar{D}_{L}=10^{(\bar{\mu}-25) / 5}$ and $\sigma_{\bar{D}_{L}}^{2}=\left(\frac{\partial \bar{D}_{L}}{\partial \bar{\mu}}\right)^{2} \sigma_{\bar{\mu}}^{2}$ (see Figs $1 \mathrm{~b}$ and 1c). Considering only SNe Ia data (Union2.1), the final sample would have only 65 SGL systems.

The constraints on the $\gamma_{0}$ and $\gamma_{1}$ parameters are obtained by evaluating the likelihood distribution function, $\mathcal{L} \propto \mathrm{e}^{-\chi^{2} / 2}$, with

$\chi^{2}=\sum_{i=1}^{92} \frac{\left.\left[D_{i}\left(\gamma_{0}, \gamma_{1}\right)-1+\frac{\bar{D}_{i L_{1}}\left(1+z_{\mathrm{s}}\right)}{\bar{D}_{i L_{\mathrm{s}}}\left(1+z_{\mathrm{l}}\right)}\right)\right]^{2}}{\sigma_{i \mathrm{obs}}^{2}}$,

\footnotetext{
${ }^{4}$ For 26 SGL systems we do not find SNe Ia (Union2.1) or GRBs obeying the (I) and (II) criteria.
}

where $D$ is given by equation (4), which depends on $\gamma_{0}$ and $\gamma_{1}$, and $\sigma_{i \text { obs }}^{2}$ stands for the statistical errors associated with the $D_{L}(z)$ from $\mathrm{SNe}$ Ia and GRBs data and to gravitational lensing observations. The $\sigma_{D}$ error is given by

$\sigma_{D}=D \sqrt{4\left(\delta \sigma_{0}\right)^{2}+(1-\gamma)^{2}\left(\delta \theta_{\mathrm{E}}\right)^{2}}$.

As discussed earlier, the statistical analyses are performed considering six SGL sub-samples, namely,

(i) $53 \mathrm{SGL}$ systems with $\sigma_{\text {ap }} \leq 250 \mathrm{~km} \mathrm{~s}^{-1}$ (low-intermediate lens masses)

(ii) $39 \mathrm{SGL}$ systems with $\sigma_{\text {ap }}>250 \mathrm{~km} \mathrm{~s}^{-1}$ (intermediate-high lens masses)

(iii) 25 SGL systems with $z_{1} \leq 0.20$ (low redshifts)

(iv) 44 SGL systems with $0.20<z_{1} \leq 0.45$ (intermediate redshifts)

(v) 23 SGL systems with $z_{1}>0.45$ (high redshifts)

(vi) Complete sample (92 SGL systems) obtained by using Union2.1 SNe Ia + GRBs

As commented by Cao et al. (2016a), elliptical galaxies with velocity dispersion smaller than $200 \mathrm{~km} \mathrm{~s}^{-1}$ may be classified roughly as relatively low-mass galaxies, while those with velocity dispersion larger than $300 \mathrm{~km} \mathrm{~s}^{-1}$ may be treated as relatively high-mass galaxies. Naturally, elliptical galaxies with velocity dispersion between 200-300 $\mathrm{km} \mathrm{s}^{-1}$ may be classified as intermediate-mass galaxies. In order to guarantee that there is enough data in each sub-sample, we consider only two sub-samples when the velocity dispersion is used as criterion.

Our results are plotted in Figs 2, 3, 4 and the best-fitting values are mentioned in Table 1. Figs 2(a), (b) and (c) show the $1 \sigma$ and $2 \sigma$ confidence regions in the $\left(\gamma_{0}-\gamma_{1}\right)$ plane considering the three $\gamma\left(z_{1}\right)$ parametrizations and SGL sub-samples defined by different lens 

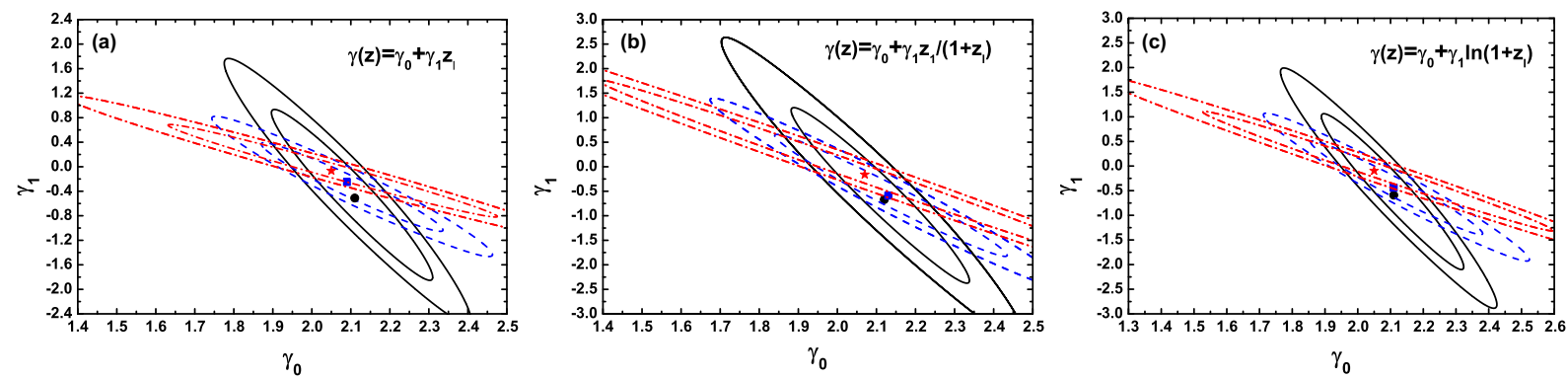

Figure 2. Figs (a), (b) and (c) show the $1 \sigma$ and $2 \sigma$ confidence contours in $\left(\gamma_{0}-\gamma_{1}\right)$ plane for all the three parametrizations. Solid black, dashed blue and dashed-dot red line contours are obtained with the SGL sub-samples having lens redshift: $z_{1} \leq 0.20,0.20<z_{1} \leq 0.45$ and $z_{1}>0.45$, respectively. The filled red star, blue square and black circle correspond to the best fits for each case.
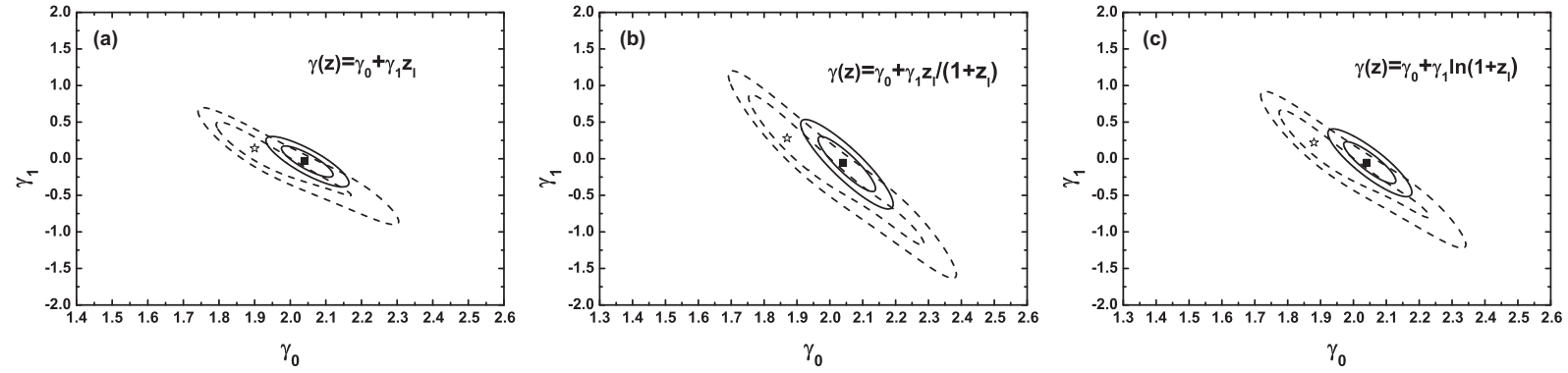

Figure 3. Figs (a), (b) and (c) show the $1 \sigma$ and $2 \sigma$ confidence contours in $\left(\gamma_{0}-\gamma_{1}\right)$ plane for all the three parametrizations. Solid black, dashed blue and dashed-dot red line contours are obtained with the SGL sub-samples with $\sigma_{\text {ap }} \leq 250 \mathrm{~km} \mathrm{~s}^{-1}$ and $>250 \mathrm{~km} \mathrm{~s}^{-1}$, respectively. Open star and filled square correspond to the best fits for each case.
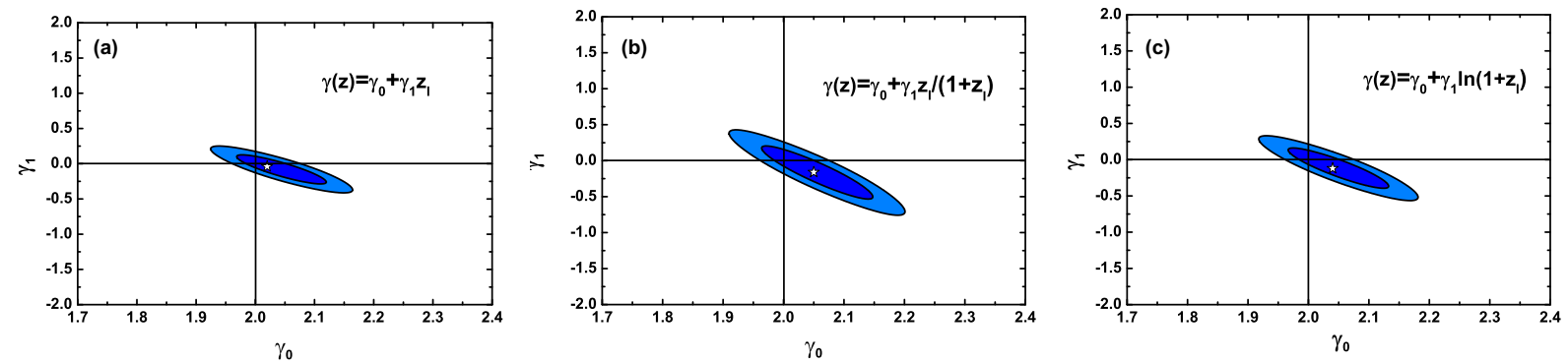

Figure 4. Figs (a), (b) and (c) show the $1 \sigma$ and $2 \sigma$ confidence contours in $\left(\gamma_{0}-\gamma_{1}\right)$ plane for all the three parametrizations with the complete sample of SGL (92 systems). The white star in each panel corresponds to the best-fitting value.

redshifts. In each panel, results obtained with the SGL sub-samples with $z_{1} \leq 0.20,0.20<z_{1} \leq 0.45$ and $z_{1}>0.45$ are shown with solid black, dashed blue and dashed-dot red lines, respectively. The filled red star, blue square and black circle correspond to the best fits for each case, respectively. From Table 1, one may see that the values are in full agreement with each other and the P1 parametrization gives the more restrictive intervals. In all cases, the SGL sub-samples in low and intermediate redshifts provide tighter regions in parameter space. The best fits of the $\gamma_{1}$ in parametrizations by using the SGL sub-sample with $z_{1}>0.45$ are closer to zero than the other SGL subsamples, but in all cases the central value is negative, suggesting a slight evolution to $\gamma\left(z_{1}\right)$.

Figs 3(a), (b) and (c) show the $1 \sigma$ and $2 \sigma$ confidence regions for the $\left(\gamma_{0}-\gamma_{1}\right)$ plane considering the three $\gamma\left(z_{1}\right)$ parametrizations and SGL sub-samples defined by different velocity dispersions of lenses. The SGL sub-samples with $\sigma_{\text {ap }} \leq 250 \mathrm{~km} \mathrm{~s}^{-1}, \sigma_{\text {ap }}>250 \mathrm{~km} \mathrm{~s}^{-1}$ are represented by the solid and dashed black lines, respectively. The filled black square and the open star correspond to the best fits for each case. Again, for each sub-sample, the regions in param- eter space depend weakly on the $\gamma\left(z_{1}\right)$ parametrization. However, by comparing the contours obtained with the SGL sub-samples in each panel, one may see that the $1 \sigma$ regions for $\left(\gamma_{0}-\gamma_{1}\right)$ are incompatible with each other. Moreover, in all cases, the best fits of the $\gamma(z)$ parametrizations by using the SGL sub-sample with $\sigma_{\text {ap }}>250 \mathrm{~km} \mathrm{~s}^{-1}$ are ruled out in $2 \sigma$ c.l. by the confidence regions of the SGL sub-sample with $\sigma_{\text {ap }} \leq 250 \mathrm{~km} \mathrm{~s}^{-1}$. Finally, the best fits of $\gamma_{1}$ are always positive when $\sigma_{\text {ap }}>250 \mathrm{~km} \mathrm{~s}^{-1}$, while for the other sub-samples are negative. These results show an interesting dependence of the $\gamma$ parameter on the mass lens.

Figs 4(a), (b) and (c) show the $1 \sigma$ and $2 \sigma$ confidence regions for the $\left(\gamma_{0}-\gamma_{1}\right)$ plane considering the 92 SGL systems, the respective luminosity distances and the three $\gamma(z)$ parametrizations. The open star corresponds to the best fits. As one may see in Table 1 , we obtain $\gamma_{0} \approx 2$ and $\gamma_{1} \approx 0$ within $1 \sigma$ c.l. for all $\gamma\left(z_{1}\right)$ parametrizations. However, the best-fitting values of $\gamma_{1}$ are slightly negative: $-0.085,-0.16$ and -0.12 for P1, P2 and $\mathrm{P} 3$ parametrizations, respectively, suggesting a mild evolution for $\gamma(z)$. 

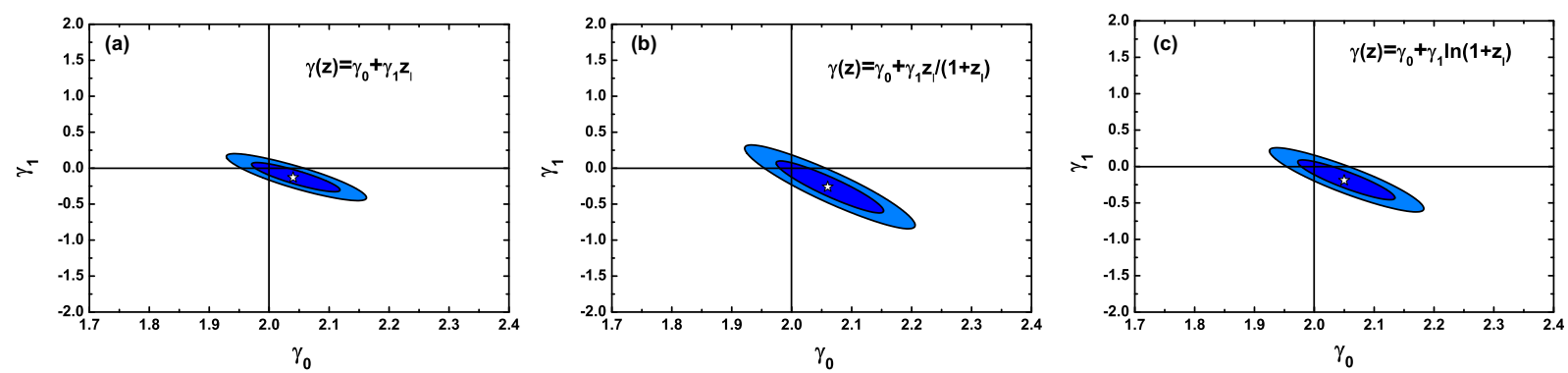

Figure 5. Figs (a), (b) and (c) show the $1 \sigma$ and $2 \sigma$ confidence contours in $\left(\gamma_{0}-\gamma_{1}\right)$ plane for all the three parametrizations by using SNe Ia from JLA compilation (Betoule et al. 2014) plus GRBs and SGL (87 systems). The white star in each panel corresponds to the best-fitting value.

We also perform an analysis by using the SNe Ia from Joint Light Analysis (JLA) compilation (Betoule et al. 2014) plus GRBs. For this case, we obtain a sub-sample with 87 SGL and the respective luminosity distances from JLA and GRBs. The JLA compilation contain 740 spectroscopically confirmed SNe Ia in the redshift range of $0.01<z<1.3$. The distance modulus of each SNe Ia further depend upon on the $\alpha, \beta$ and $M$ as mentioned in Section 3.2 for Union2.1 compilation. In recent works (Nielsen, Guffanti \& Sarkar 2016; Evslin 2016; Nunes, Pan \& Saridakis 2016; Li et al. 2016b), it has been observed that $\alpha$ and $\beta$ act like global parameters, whatever prior cosmological model one chooses to find the distance modulus of each SNe. So we fix the values of $\alpha$ and $\beta$ as given by Betoule at al. (2014). The results obtained with this data set ( 87 data points) are plotted in Figs 5(a), (b) and (c). They show the $1 \sigma$ and $2 \sigma$ confidence regions in the $\left(\gamma_{0}-\gamma_{1}\right)$ plane for all the three $\gamma(z)$ parametrizations. The open star corresponds to the best fits. As one may see in Table 1 (last line), we obtain again $\gamma_{0} \approx 2$ and $\gamma_{1} \approx 0$ within $1 \sigma$ c.l. for all $\gamma\left(z_{l}\right)$ parametrizations. The $1 \sigma$ and $2 \sigma$ c.l. regions are in full agreement with those from Union2.1 plus GRBs (Fig. 4). However, the best-fitting values of $\gamma_{1}$ are more negative, we obtain: -0.13 , -0.26 and -0.19 for $\mathrm{P} 1, \mathrm{P} 2$ and $\mathrm{P} 3$ parametrizations, respectively, reinforcing a mild evolution for $\gamma(z)$.

\subsection{Comparing results}

It is interesting to compare our results by using the 92 and 87 SGL systems (last two lines in Table 1) with previous ones where $\gamma_{0}$ and $\gamma_{1}$ were constrained by adopting the P1 parametrization and different cosmological model in analyses. For instance:

(i) Cao et al. (2015a) used exclusively 118 SGL systems and they found $\gamma_{0}=2.13_{-0.12}^{+0.07}$ and $\gamma_{1}=-0.09 \pm 0.17$ in a $\omega \mathrm{CDM}$ model, and $\gamma_{0}=2.14_{-0.10}^{+0.07}$ and $\gamma_{1}=-0.10 \pm 0.18$ in a $\omega(z) \mathrm{CDM}(\mathrm{CPL}$ model) framework. In both cases the matter density parameter was fixed $\left(\Omega_{M}=0.315\right)$ based on the Planck results (Ade et al. 2014).

(ii) Cui, Li \& Zhang (2017), more recently, by using the SGL observations in combination with other cosmological observations (BAO, CMB and $H(z)$ data), considered some simple dark energy models, such as $\omega \mathrm{CDM}$, the holographic dark energy model (Li 2004) and the Ricci dark energy model (Gao et al. 2009). Briefly, these authors derived $\gamma_{0} \approx 2.10$ (with the uncertainty around 0.04 0.05 ) and $\gamma_{1} \approx-0.06$ (with the uncertainty around 0.1 ).

(iii) Li et al. (2016a) considered SGL observations plus BAO measurements and found the following values: $\gamma_{0}=2.094_{-0.056}^{+0.053}$ and $\gamma_{1}=-0.053_{-0.102}^{+0.103}$ in a $\Lambda \mathrm{CDM}$ model, $\gamma_{0}=2.088_{-0.056}^{+0.055}$ and $\gamma_{1}=-0.054_{-0.02}^{+0.104}$ in a $\omega \mathrm{CDM}$ model, $\gamma_{0}=2.087_{-0.056}^{+0.055}$ and $\gamma_{1}=-0.055 \pm 0.105$ in a $\omega(z) \operatorname{CDM}$ (CPL model), $\gamma_{0}=2.087_{-0.054}^{+0.052}$ and $\gamma_{1}=-0.052_{-0.102}^{+0.104}$ in a Ricci dark energy model and, finally, $\gamma_{0}=2.074_{-0.051}^{+0.050}$ and $\gamma_{1}=-0.047_{-0.102}^{+0.101}$ in a Dvali-GabadadzePorrati brane world model.

As one may see, these previous results for $\gamma_{0}$ are in agreement with the present work within $1 \sigma$ c.l., although they show a departure from $\gamma_{0}=2$ at least at $1 \sigma$ c.l.. On the other hand, in all cases $\gamma_{1} \approx$ 0 is allowed within $1 \sigma$ c.l., indicating that a significant time varying $\gamma$ is not supported by the current observations.

Finally, we also compare our results with those from Cao et al. (2016a). These authors used the complete SGL sample (118 points) from Cao et al. 2015a, the flat $\Lambda$ CDM model (Ade et al. 2014), the P1 parametrization and 6 sub-samples similar to those considered in the present work. The sub-samples consist of: 25, 80 and 13 SGL systems with $\sigma_{\text {ap }} \leq 200 \mathrm{~km} \mathrm{~s}^{-1}, 200<\sigma_{\text {ap }} \leq 300 \mathrm{~km} \mathrm{~s}^{-1}$ and $\sigma_{\text {ap }}>300 \mathrm{~km} \mathrm{~s}^{-1}$, respectively, and, 25, 65 and 80 SGL systems with $z_{1} \leq 0.20,0.20<z_{1} \leq 0.50$ and $z_{1}>0.50$, respectively. The main points are:

(i) Results obtained by using the 118 SGL systems $\left(\gamma_{0}=2.132 \pm 0.055, \gamma_{1}=-0.067 \pm 0.119\right)$ are in full agreement with the present work when the 92 SGL systems are used, but their result for $\gamma_{0}$ value is incompatible with 2.0 at least for $1 \sigma \mathrm{c} .1$. The $\gamma_{1}$ value is in full agreement with ours (within $1 \sigma$ c.l.).

(ii) Cao et al. (2016a) results from the sub-samples defined by the different lens redshifts are in full agreement with ours (within $1 \sigma$ c.l.).

(iii) By using the lens velocity dispersions as criterion, similar behaviour for $\gamma(z)$ is found if one compares our SGL sub-sample of low velocity dispersions with theirs of low and intermediate velocity dispersions. However, we obtain for the $\gamma_{1}$ parameter a best-fitting value more positive (Cao et al. 2016a found for this case $\left.\gamma_{1}=-0.047\right)$. The source of this difference may lie in the samples used in the analyses or in the cosmological model considered.

Naturally, our error bars are larger since we have performed the analyses without using a specific cosmological model.

\section{CONCLUSION}

Knowing the exact profile of mass distribution for strong gravitational lensing systems is very important in order to use this phenomenon as a precise cosmological tool. In analyses with timedelay distance, for instance, different assumptions lead to different $H_{0}$ estimates. The simplest model used frequently in strong gravitational lensing observations is the singular isothermal sphere (the SIS model). However, it has been changed by a power-law mass distribution $\left(\rho \propto r^{-\gamma}\right)$ since recent studies in elliptical galaxies have shown a non-negligible scatter from the SIS model. A crucial point in the power-law mass distribution is to know if the $\gamma$ parameter 
varies with redshift, since this fact is linked to massive galaxies growth process.

In this paper we propose a new method to access a possible $\gamma$ variation. Our theoretical framework was based on two assumptions: a flat universe and the validity of cosmic distance duality relation. No specific cosmological model was used. We also considered three $\gamma(z)$ parametrizations, namely: (P1) $\gamma\left(z_{1}\right)=\gamma_{0}+\gamma_{1} z_{1}$, (P2) $\gamma\left(z_{1}\right)=\gamma_{0}+\gamma_{1} z_{1} /\left(1+z_{1}\right)$ and (P3) $\gamma\left(z_{1}\right)=\gamma_{0}+\gamma_{1} \ln \left(1+z_{1}\right)$. By using 92 strong gravitational lensing observations plus $\mathrm{SNe}$ Ia (Union2.1) and GRBs, we find no significant $\gamma(z)$ evolution. However, in all cases the best-fitting values for the $\gamma_{1}$ parameter were found to be negative (except in the sub-sample $\sigma_{\text {ap }}>250 \mathrm{~km} \mathrm{~s}^{-1}$ ), indicating a mild evolution for $\gamma\left(z_{1}\right)$. Although less restrictive, our results are also in full agreement with recent results from other cosmological model dependent methods (see Section 4). The lenses and sources of the SGL systems lie in the redshift range $0.073 \leq$ $z_{1} \leq 0.783$ and $0.0196 \leq z_{\mathrm{s}} \leq 3.59$. The mild evolution was reinforced when we considered a sub-sample with 87 SGL systems and the respective luminosity distances obtained from the JLA SNe Ia compilation and GRBs.

We also considered the analyses by using sub-samples of the SGL systems defined by different lens redshifts and velocity dispersions plus SNe Ia (Union2.1) and GRBs. The results obtained from subsamples with $z_{1} \leq 0.20,0.20<z_{1} \leq 0.45$ and $z_{1}>0.45$ (where $z_{1}$ is the lens redshift) are in full agreement each other. On the other hand, we found that the best fits for the SGL sub-sample with $\sigma_{\text {ap }}>250 \mathrm{~km} \mathrm{~s}^{-1}$ are ruled out in $2 \sigma$ c.l. by the confidence regions of the SGL sub-sample with $\sigma_{\text {ap }} \leq 250$. Moreover, the best fits of the SGL sub-sample with $\sigma_{\text {ap }}<250 \mathrm{~km} \mathrm{~s}^{-1}$ are negative, while for the other SGL sub-sample are positive. Our results reinforce the need of treating galaxies with low and high velocity dispersions separately.

In the near future, it is expected that several surveys (EUCLID mission, Pan-STARRS, LSST, JDEM) discover thousands of strong lensing systems. Then by applying this method along with a bigger sample, more stringent limits on the parameters $\gamma_{0}$ and $\gamma_{1}$ can be obtained. Besides, as an interesting extension of the present paper, one may check the consequences of relaxing the rigid assumption that the stellar luminosity and total mass distributions follow the same power law. Also, it would be interesting in the future to apply this method with the inclusion of other sources at cosmological distances, like powerful radio sources (Gurvitz 1994; Gurvitz, Kellermann \& Frey 1999; Jackson 2004; Jackson \& Jannetta 2006; Cao et al. 2015b, 2017b). The inclusion of these high redshift sources further would corroborate or even contradict our present conclusions.

\section{ACKNOWLEDGEMENTS}

RFLH acknowledges financial support from Conselho Nacional de Desenvolvimento Científico e Tecnológico (CNPq) and UEPB (No. 478524/2013-7, 303734/2014-0). SHP is grateful to CNPq, for financial support (No. 304297/2015-1, 400924/2016-1). The authors are grateful to the referee for very constructive comments.

\section{REFERENCES}

Ade P.A.R., Planck Collaboration XVI et al., 2014, A\&A, 571, A16 Auger M. W., Treu T., Bolton A. S., Gavazzi R., Koopmans L. V. E., Marshall P. J., Moustakas L. A., Burles S., 2010, ApJ, 724, 511

Barnabe M., Czoske O., Koopmans L. V. E., Treu T., Bolton A. S., 2010, MNRAS, 415, 2215
Bartelmann M., Schneider P., 2001, Phys. Rep., 340, 291

Bassett B. A., Kunz M., 2004, Phys. Rev. D, 69, 101305

Betoule M. et al., 2016, A\&A, 568, A22

Biesiada M., 2006, Phys. Rev. D, 73, 023006

Cao S., Liang N., 2011, Res. Astron. Astrophys., 11, 1199

Cao S., Zhu Z.-H., 2012, A\&A, 538, A43

Cao S., Pan Y., Biesiada M., Godlowski W., Zhu Z.-H., 2012, JCAP, 03, 016

Cao S., Biesiada M., Gavazzi R., Piórkowska A., Zhu Z.-H., 2015a, ApJ, 806,185

Cao S., Biesiada M., Zheng X., Zhu Z.-H., 2015b, ApJ, 806, 66

Cao S., Biesiada M., Yao M., Zhu Z.-H., 2016a, MNRAS, 481, 2192

Cao S., Biesiada M., Zheng X., Zhu Z.-H., 2016b, MNRAS, 457, 281

Cao S., Li X., Biesiada M., Xu T., Cai Y., Zhu Z.-H., 2017, ApJ, 835, 92

Cao S., Biesiada M., Jackson J., Zheng X. Zhao Y., Zhu Z.-H., 2017, JCAP, 02,012

Coe D., Moustakas L., 2009, ApJ, 706, 45

Cui J-L., Li H-L., Zhang X., 2017, Sci. China-Phys. Mech. Astron., 60, 080411

Cunha J. V., Marassi L., Santos R. C., 2007, Int. J. Mod. Phys. D, 16, 403

Demianski M., Piedipalumbo E., Sawant D., Amati L., 2017, A\&A, 598, A112

Ellis G. F. R., 1971, in Sachs R. K., ed., Proc. Int. School Phys. Enrico Fermi, Relativistic Cosmology, Vol. XLVII. Academic Press, New York, p. 104

Etherington I. M. H., 1933, Phil. Mag, 15, 761

Evslin J., 2016, Phys. Dark Univ., 14, 57

Futamase T., Yoshida S., 2001, Prog. Theor. Phys., 105, 887

Gao C., Wu F. Q., Chen X., Shen Y.-G., 2009, Phys. Rev. D, 79, 043511

Grillo C., Lombardi M., Bertin G., 2008, A\&A, 477, 397

Gurvitz L. I., 1994, ApJ, 425, 442

Gurvitz L. I., KellermannK. I., Frey S., 1999, A\&A, 342, 378

Guy J. et al., 2007, A\&A, 466, 11

Holanda R. F. L., 2016, Astropart. Phys., 84, 78

Holanda R. F. L., Lima J. A. S., Ribeiro M. B., 2011, A\&A, 528, L14

Holanda R. F. L., Busti V. C., Alcaniz J. S., 2016, JCAP, 02, 054

Holanda R. F. L., Busti V. C., Lima F. S., Alcaniz J. S., 2016, preprint (arXiv:1611.09426)

Jackson J. C., 2004, JCAP, 11, 7

Jackson J. C., Jannetta A. L., 2006, JCAP, 11, 2

Jee I., Komatsu E., Suyu S. H., 2015, JCAP, 11, 033

Kochanek C. S., Schneider P., Wambsganss J., 2004, in Meylan G., Jetzer P., North P., eds, Part 2 Gravitational Lensing: Strong, Weak \& Micro Springer-Verlag, Berlin

Koopmans L. V. E., 2005, in Mamon G. A. et al., eds, Proc. XXIst IAP Coll., Mass Profiles and Shapes of Cosmological Structures. EDP Sciences, France, p. 161

Koopmans L. et al., 2009, ApJ, 703, L51

Li M., 2004, Phys. Lett. B, 603, 1

Li X. L., Cao S., Zheng X. G., Li S., Biesiada M., 2016a, Res. Astron. Astrophys, 16, 8

Li Z., Wang G. J., Liao K., Zhu Z. H., 2016b, ApJ, 833, 240

Liao W., Li Z., Cao S., Biesiada M., Zheng X., Zhu Z.-H., 2016, ApJ, 822, 74

Longair M. S., 1998, Galaxy Formation. Springer-Verlag, Berlin

Mantz A., Allen S. W., Morris R. G., Rapetti D. A., Applegate D. E., Kelly P. L., von der Linden A., Schmidt R. W., 2014, MNRAS, 440, 2077

Mitchell J. L., Keeton C. R., Frieman J. A., Sheth R. K., 2005, ApJ, 622, 81

Nielsen J. T, Guffanti A., Sarkar S., 2016, Nature Sci. Rep., 6, 35596

Nunes R. C., Pan S., Saridakis E. N., 2016, Phys. Rev. D, 94, 023508

Ofek E. O., Rix H.-W., Maoz D., 2003, MNRAS, 343, 639

Paraficz D., Hjorth J., 2009, A\&A, 507, L49-52

Rana A., Jain D., Mahajan S., Mukherjee A., 2016, JCAP, 07, 026

Refsdal S., 1964, MNRAS, 128, 295

Ruff A. J., Gavazzi R., PMarshall. J., Treu T., Auger M. W., Brault F., 2011, ApJ, 727, 96

Saha P., Coles J., Maccio A. V., Williams L. L. R., 2006, ApJ, 650, L17 
Schneider P., Ehlers J., Falco E. E., 1992, Gravitational Lenses, SpringerVerlag, Berlin

Schwab J., Bolton A. S., Rappaport S. A., 2010, ApJ, 708, 750

Sonnenfeld A., Treu T., Gavazzi R., Suyu S. H., Marshall P. J., Auger M. W., Nipoti C., 2013, ApJ, 777, 98

Suyu S. H., Marshall P. J., Auger M. W., Hilbert S., Blandford R. D., Koopmans L. V. E., Fassnacht C. D., Treu T., 2010, ApJ, 711, 201
Suyu S. H. et al., 2013, ApJ, 766, 70

Suzuki N. et al., 2012, ApJ, 746, 85

Treu T., 2010, ARA\&A, 48, 87

Zhu Z.-H., Hu M., Alcaniz J. S., Liu Y.-X., 2008, A\&A, 483, 15

This paper has been typeset from a $\mathrm{T}_{\mathrm{E}} \mathrm{X} / \mathrm{LAT}_{\mathrm{E}} \mathrm{X}$ file prepared by the author. 